\title{
A Case Report on Purple Urine Bag Syndrome
}

\author{
Kavya Nedamanuru*, Sai Jyothi Dampetla, Divya Gopineni
}

Department of Pharmacy Practice, Sri Padmavathi School of Pharmacy, Tiruchanoor, Tirupathi, Andhra Pradesh, INDIA.

\begin{abstract}
Discoloration of urine is not uncommonly encountered in clinical practice and may indicate a significant pathology. However, the majority of instances are benign and occur as the result of trauma to the urological system during procedures or ingestions of substances such as medication or food. This phenomenon is known as the purple urine bag syndrome. It is associated with urinary tract infections occurring in catheterised patients, generally elderly females with significant co-morbidities and constipation. The purple discoloration is most often due to the presence of indigo and indirubin pigments which are the metabolites of tryptophan. In this article, we present a case of elderly man with this rare and interesting phenomenon after insertion of an indwelling catheter. The purple urine disappeared after antibiotic therapy.
\end{abstract}

Key words: Purple urine bag syndrome, Tryptophan, Catheterisation, Antibiotic therapy.

\section{INTRODUCTION}

Purple urine bag syndrome (PUBS) is a rare disease entity first reported in 1978 and is signified by an alarming purple discoloration of the urine usually seen in women and chronically debilitated patients with long term indwelling urinary catheter. ${ }^{1}$ PUBS can be distressing for patients, family members and healthcare workers who are unaware of this association. This condition is often associated with urinary tract infection. The purple colour is due to the compounds indigo and indirubin, which are metabolic degeneration products of tryptophan released at multiple stages by bacterial enzymes in the intestine and urine. ${ }^{2}$

The condition usually affects elderly women. Most patients are bedridden, and many have cognitive impairment. Constipation has been reported as a frequent symptom in these patients. Almost all PUBS patients are catheterized due to significant disability, and are chair-bound or bed-bound. Discoloration of the urine bag appears after about 2-3 months of catheterization, and will be shown after more than 1 year. ${ }^{3}$ PUBS can be associated with significant morbidity and mortality, but PUBS itself is a benign condition. Treatment should be aimed at the underlying medical problem rather than at PUBS itself. ${ }^{4}$ Therefore, clinician should early diagnose the condition and prescribe appropriate Anti-biotic regimen to reduce the morbidity and mortality of patient.

\section{CASE REPORT}

A 72-year-old elderly male with long standing history of hypertension, coronary artery disease and recent history of ischemic stroke presented with difficulty in swallowing, weakness of left upper and lower limbs, inability to speak and was bedridden for 6 months. As he was unable to move, Foley's catheter was inserted to the bladder to drain urine since the attack of Hemiplegia. Purple urine bag was found after re-admission to the hospital. After 3 days of admission, haematuria was observed. Urinary catheter was removed immediately, and condom catheter was inserted to the patient on referring to Urology Department. Colour of urine in the bag was found to be purple. The urinalysis showed the presence of 8-9 pus cells and 3-4 epithelial cells. The Antibiotic therapy was started with Intravenous
DOI: 10.5530/ijopp.10.4.59

Address for correspondence: Kavya Nedamanuru, Department of pharmacy practice, Pharm. D 5th year, Department of Pharmacy Practice, Sri Padmavathi School of Pharmacy, Tiruchanoor, Tirupathi-517502, Andhra Pradesh, INDIA. Phone no: +7993170355 Email Id: kavyanedamanuru@ gmail.com

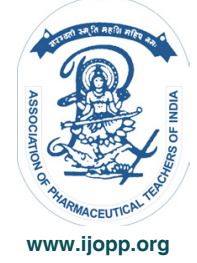


injection Ciprofloxacin 200mg twice daily. After 3 days, Piperacillin-Tazobactam at a dose of 4.5 gram thrice daily was added to therapy and continued for next 3 days. The purple urine was disappeared, and the following urinalysis was sterile. He was discharged in stable condition.

\section{DISCUSSION}

PUBS is an uncommon phenomenon in which purple discoloration of catheter bag is due to the presence of blue pigment indigo and red pigment indirubin. The proposed mechanism for pigment production is through altered metabolism of tryptophan. ${ }^{2}$ Owing to altered gut motility or intestinal bacterial overgrowth associated with chronic constipation, tryptophan is exposed to intestinal flora before it can be absorbed. Bacteria deaminate tryptophan to yield indole that is absorbed into portal circulation and converted in liver to indoxyl sulphate after conjugation. ${ }^{5,6}$ Levels of indoxyl sulphate in the urine are usually elevated above the range. Urine bacteria with indoxyl sulphatase activity convert indoxyl sulphate into indoxyl. In alkaline environment, indoxyl turns into indigo and indirubin pigments that combine with catheter tubing to give purple appearance. PUBS have been associated with multiple pathogens including Pseudomonas, Morgenella, Proteus, Providencia, Klebsiella, and Enterobacter species. ${ }^{7,8} \mathrm{Gram}$-negative bacteria producing phosphatase/ sulphatase are important in pathogenesis. Here patient was started with Fluoroquinolone i.e., Ciprofloxacin and Penicillin derivative i.e., PiperacillinTazobactum (broad spectrum antibiotics) for gramnegative bacterial infection as per hospital antibiotic policy and sensitivity pattern.

\section{CONCLUSION}

PUBS is a relatively rare clinical phenomenon but has higher incidence in geriatric patients, with female gender, dementia, alkaline urine, constipation, chronic catheterisation with indwelling catheters, high bacterial loads in urine, and chronic kidney disease serving as risk factors for its development. Although PUBS is usually harmless and does not require treatment, it is directed at the underlying UTI by antibiotics as well as by removing an unnecessarily inserted urinary catheter. Proper care of urinary catheters and control of constipation are important measures to avoid this condition. Good urologic hygiene by good care and frequent change of urinary catheters may help in preventing this uncommon entity.

\section{ACKNOWLEDGEMENT}

We thank our guide Mrs. G. Divya, physicians and PGs at SVRRGGH Tiruapti.

\section{ABBREVIATIONS USED}

PUBS: Purple Urine Bag Syndrome; UTI: Urinary Tract Infection.

\section{CONFLICT OF INTEREST}

There are no conflicts of interest.

\section{REFERENCES}

1. Makkar V, Mann A. Purple urine bag syndrome: a case report. Int J Med Sci and Pub Hea. 2015; 4(8):1157-8.

2. Hadano $Y$, Shimizu $T$, Shimon $T$, Inous $T$, Sorano $S$. An update on purple urine bag syndrome. Int J Gen Med. 2012;5:707-10.

3. Harun Ns, Nainar Sk, Chong VH. Purple urine bag syndrome: A rare and interesting phenomenon. South Med J. 2007;100(10):1048-50.

4. Foot C, Fraser JF. Uroscopic Rainbow: Modern Matula medicine. Postgrad Med J 2006;82(964):126-9.

5. Barlow GB, Dickson JA. Purple urine bags. Lancet. 1978;311(8057):220-1.

6. Dealler SF, Belfield PW, Bedford M, Whitley AJ, Mulley GP.Purple urine bags. J Urol. 1989;142(3):769-70.

7. Arnold W. King George IIl's Urine and Indigo Blue. Lancet. 1996;347(9018):1811-3.

8. Jones RA, Deacon HJ, Allen SC. Two cases and a short discussion of purple urine bag syndrome. CME Geriatr Med. 2003;5:84-7. 